\title{
Time-frequency representation based on time-varying autoregressive model with applications to non-stationary rotor vibration analysis
}

\author{
LONG ZHANG ${ }^{1}$, GUOLIANG XIONG ${ }^{2, *}$, HESHENG LIU ${ }^{3}$, \\ HUIJUN ZOU ${ }^{1}$ and WEIZHONG GUO ${ }^{1}$
}

\author{
${ }^{1}$ Mechanical Engineering School, Shanghai Jiao Tong University, Shanghai \\ 200240, P.R. China \\ ${ }^{2}$ School of Mechanical and Electronical Engineering, East China Jiaotong \\ University, Nanchang Jiangxi 330013, P.R. China \\ ${ }^{3}$ Department of Physics, Shangrao Normal University, Shangrao Jiangxi 334001, \\ P.R. China \\ e-mail: longzh@126.com; lgxcxx@ecjtu.jx.cn
}

MS received 6 June 2008; revised 2 December 2009; accepted 17 January 2010

\begin{abstract}
A parametric time-frequency representation is presented based on timevarying autoregressive model (TVAR), followed by applications to non-stationary vibration signal processing. The identification of time-varying model coefficients and the determination of model order, are addressed by means of neural networks and genetic algorithms, respectively. Firstly, a simulated signal which mimic the rotor vibration during run-up stages was processed for a comparative study on TVAR and other non-parametric time-frequency representations such as Short Time Fourier Transform, Continuous Wavelet Transform, Empirical Mode Decomposition, Wigner-Ville Distribution and Choi-Williams Distribution, in terms of their resolutions, accuracy, cross term suppression as well as noise resistance. Secondly, TVAR was applied to analyse non-stationary vibration signals collected from a rotor test rig during run-up stages, with an aim to extract fault symptoms under non-stationary operating conditions. Simulation and experimental results demonstrate that TVAR is an effective solution to non-stationary signal analysis and has strong capability in signal time-frequency feature extraction.
\end{abstract}

Keywords. Non-stationary signal; time-varying autoregressive model; TVAR; fault diagnosis; rotor.

\section{Introduction}

Production machinery is the fundamental of manufacturing engineering. Condition monitoring and fault diagnosis of those equipments have been gaining importance because of the need

*For correspondence 
to increase the reliability and to decrease the possibility of production loss due to machine breakdown (Zhan et al 2006; Zhan \& Jardine 2005; Wang \& Kootsookos 1998).

Vibration signal analysis is a key issue to condition monitoring and fault diagnosis (Gupta 1997). The Fast Fourier Transform (FFT) of vibration signals is the most conventionally adopted method, which is based on the assumption that the signals under consideration were stationary and cyclic. In most cases, such an assumption however cannot be justified due to the fact that inherent nonlinearity of equipments, occurrence of faults, fluctuating loads and operating speed will render the statistics of the measured signals change over a time. It implies that most realistic vibration signals are usually non-stationary and non-cyclic. As such, processing such signals by FFT will mostly result in an ambiguous frequency spectrum which brings difficulties to the extraction of condition indicating information. Hitherto, many efforts have been made to develop techniques for processing non-stationary signals like time-frequency representation (TFR) (Zhan \& Jardine 2005; Dowling 1993; Gao \& Yan 2006; Conforto \& D'alessio 1999). TFR is able to produce an overall view of the behaviour of non-stationary signals by means of so-called time-varying spectrum. Such a spectrum is defined in a time-frequency space and represents the evolution of signal power as a function of both time and frequency (Zhan \& Jardine 2005; Conforto \& D'alessio 1999). In general, TFR falls into two classes, non-parametric and parametric one (Conforto \& D'alessio 1999; Wang et al 2008). The most extensively exploited non-parametric methods include Short Time Fourier Transform (STFT), Wigner-Ville Distribution (WVD), Choi-Williams Distribution (CWD), Wavelet Transform (WT), Empirical Mode Decomposition (EMD) and their enhanced derivations, etc. STFT is essentially composed of piece-wise FFTs, which assumes that the signal of interest is locally stationary. According to the 'Uncertainty principle', the STFT is unlikely to obtain fine resolutions in both time and frequency domains simultaneously. WVD performs well on signals of single component. For multi-component signals, the performance of the WVD will degrade detrimentally because the spurious peaks arising from cross terms will confuse the visual interpretation of its time frequency spectrum. CWD is an improvement of the WVD intended to supress the 'cross terms'. But any attempt to reduce cross terms is necessarily based on the loss in time and frequency resolution (Gao \& Yan 2006; Rajagopalan et al 2005).

WT has two forms of implementation, of which one is discrete WT (DWT) and the other is continuous WT (CWT). DWT performs like a dyad filter, by which the inspected signal is split into two parts, e.g. details of high frequency and approximations of low frequency. DWT provides a convenient way for filter design as well as for signal decomposition and reconstruction, so it has been extensively employed for vibration signal processing as evidenced by numerous literatures (Ebersbach et al 2006; Paya et al 1997; Peng \& Chu 2004; Wang \& McFadden 1996). In spite of the DWT's wide popularity, its inherent deficiencies should not be neglected such as border distortion and energy leakage (Peng et al 2009). The border distortion which is also known as boundary effect, refers to the computation problem induced by insufficient data points occurring at both the beginning and the end of the analysed signal. Such a problem makes the interpretation of the DWT results difficult and can only be alleviated to certain extent by zero-padding or symmetric extending to the signals (Peng et al 2009). On the other hand, energy leakage due to the overlapping between the two filters of the DWT leads to a great information redundancy. One approach to relieve energy leakage is to increase the vanish moment of the wavelet function, which however aggravates the computation burden. Meanwhile, the division of frequency band by the DWT is too coarse to offer an exact characterization of mechanical vibration signals. As such, the CWT has gaining a lot of applications in vibration signal analysis for fault diagnosis (Chiementin 
et al 2007; Gryllias \& Antoniadis 2009; Rubini \& Meneghetti 2001; Shi et al 2004; Nagaraju et al 2009) due to the fact that CWT is able to decompose the signals into a fine divided frequency grid. However, this advantage is based on the expense of computational efficiency. Another problem with the CWT is the determination of the range of scales. In addition, a common problem shared by the DWT and CWT is how to select a suitable wavelet basis function. At present, selecting method depends mainly on the waveform similarity between the signature required to extract and the wavelet basis. However, this method does not take wavelet frequency domain property into consideration ( $\mathrm{Li}$ et al 2007). WT also suffers from the 'Uncertainty Principle', thereby unable to obtain high resolutions within both low and high frequency regions simultaneously. Owing to the above reasons, the applications of the WT have not yet achieved a standard status compared with the FFT.

EMD is a relatively recent approach for nonlinear and non-stationary signal processing and has been attracting great attentions (Loutridis 2006; Rai \& Mohanty 2007; Rouillard \& Sek 2005). Unlike WT, EMD does not require the selection of basis function and the range of scales, so it is recognized as an adaptive method. Additionally, EMD has good computational efficiency and does not involve the concept of the frequency resolution and the time resolution. On account of above merits, EMD has found applications in vibration signal processing and fault diagnosis, but it still suffers some serious deficiencies like followings. At first. the physical meaning of the intrinsic mode function (IMF) resulting from the EMD sifting process and the EMD formalism are still open questions. This make its users feel not safe with its results. Secondly, there is an unavoidable deficiency-ripple phenomenon in its estimated frequency, which would mislead our analysis. Further, EMD reported is unable to correctly decompose signals of which the components are very close with each other in frequency. And usually, the first obtained IMF covers too broad a frequency range such that the monocomponent condition cannot be ensured for the first IMF (Peng et al 2005a; Peng et al 2005b). This was also confirmed in the present work. Peng et al (2005a and 2005b) proposed a signal preprocessing method for EMD by using WT, but the introduction of the linear operation of WT will compromise the adaptive property of EMD. Until now, researches on EMD are mainly limited to its applications and the improvements of its techniques such as stopping criterion of sifting process (Cheng et al 2006), end effect (Cheng et al 2007; Qi et al 2007), fitting method (Chen et al 2006), mode mixing (Li et al 2005). The researches on the theoretical foundation of the EMD are still very much lacking.

In view of the aforementioned deficiencies of non-parametric time-frequency representations, we resort to parametric ones in order to extract features from non-stationary vibration signals. Time-varying autoregressive model (TVAR) has a similar structure to that of conventional AR model, but TVAR has time-varying coefficients. TVAR inherits all the advantages of the AR in terms of high accuracy, good resolution, sharp peak, and less spurious component even for short data record. So far, TVAR has found its ways in many fields, such as audio and speech processing (Sodsri 2003; Hall et al 1983; Härmä et al 2000), vehicle type identification (Eom 1999), vibration signal processing for condition monitoring and fault diagnosis (Zhan et al 2006; Zhan \& Jardine 2005; Conforto \& D'alessio 1999; Wang et al 2008; Poulimenos \& Fassois 2006).

For a data record of $N$ samples, the conventional AR model of order $p$ has altogether $p$ coefficients, while in the case of TVAR with the same order, there are a total number of $N \times p$ coefficients due to its instantaneous attribute. Hence, TVAR gives rise to computational complexity. According to the fashion of the time-varying coefficients evolution over a time, TVAR is possible to be categorized into two classes, i.e. adaptive method and basis function approach (Conforto \& D'alessio 1999; Wang et al 2008; Poulimenos \& Fassois 2006; 
Sodsri 2003). The adaptive method is characterized by low parsimony, namely a total number of $N \times p$ coefficients need to be estimated. Moreover, it's only capable of tracing signals with weak or medium non-stationary dynamics (Poulimenos \& Fassois 2006; Sodsri 2003). In the case of the basis function approach, each of its time-varying coefficients is expressed as a weighted combination of a predetermined family of $m+1$ basis functions. Thus a total number of $(m+1) \times p$ parameters need to be identified usually far less than $N \times p$, resulting in an economical modelling with high computational efficiency. Furthermore, according to theoretical and experimental proofs in many literatures (Poulimenos \& Fassois 2006; Sodsri 2003; Eom 1999), TVAR based on basis function method is capable of tracing strong nonstationary signals. Hence, this model is focused in the present study.

Model coefficient estimation and order determination are two important aspects in TVAR modelling. In the 1970s, there are already some initial works on TVAR. Grenier (1983) extended some known methods of conventional AR to TVAR such as the Levinson AR method, Cadzow ARMA, Burg as well as Prony methods, TVAR gradually gained attention from some communities like signal processing to mechanical engineering. Owing to much more coefficients to be estimated in TVAR than in conventional AR, it will possibly lead to computational complexity and matrix singularity if the covariance and correlation functions were used as in conventional AR. Meanwhile, in view of the superior performance of artificial neural networks (ANNs) in system identification and prediction as well as ANNs' power in parallel computing, Tian et al (1997) exploited ANNs to AR model identification. In the present work, we made attempts to adopt ANNs for the estimation of TVAR model coefficients.

Model order plays a critical role in TVAR. At present, model order determination criteria such as AIC, BIC FPE and MDL are all designed for conventional AR or ARMA. In contrast, the criteria for TVAR are very rare. Tommaso \& Irene (2001), Chen (2005) and Chen et al (2007) proposed some new order determination method for ARMA based on genetic Algorithms (GAs). In Tommaso \& Irene (2001), GAs were used to simultaneously determine the structure and order of ARMA. In Chen (2005) and Chen et al (2007), different criteria and methods of optimal order search were proposed based on GAs respectively. In this work, a maximum likelihood criterion and GA-based order search were proposed for TVAR.

Model determination of TVAR by ANNs and GAs is discussed in section 2. Section 3 includes simulation along with the comparison between TVAR and other time-frequency representations such as STFT, WVD, CWD, WT and EMD. In section 4, the experimental set-up is demonstrated and results are discussed. Conclusions are provided in section 5 .

\section{TVAR model description and identification}

\subsection{Model description}

Let $x(n)$ be a zero-mean non-stationary time series with $N$ samples. The time-varying autoregressive (TVAR) model of $x(n)$ can be defined as

$$
x(n)=-\sum_{i=1}^{p} a_{i}(n) x(n-i)+\sigma(n),
$$

where $\left\{\alpha_{i}(n)\right\}$ are the time-varying model coefficients. The term $\sigma(n)$ is a zero-mean white Gaussian noise with variance $\delta^{2}>0$ when the model is correctly established. The index $n$ denotes time instant, and $p$ represents the model order. 
One-step-ahead (OSA) prediction of the model can be determined by

$$
\hat{x}(n)=-\sum_{i=1}^{p} a_{i}(n) x(n-i) .
$$

As mentioned in the 'Introduction' part of this paper, the time-varying coefficients $\left\{\alpha_{i}(n)\right\}$ of TVAR model could be expanded as a weighted sum of a family of known basis functions $\left\{f_{j}(n), j=0,1, \ldots, m\right\}$,

$$
a_{i}(n)=\sum_{j=0}^{m} a_{i j} f_{j}(n) .
$$

A variety of basis functions $\left\{f_{j}(n)\right\}$ are available for TVAR (Poulimenos \& Fassois 2006; Sodsri 2003; Eom 1999; Hall et al 1983; Harma \& Juntunen 2000), such as Fourier basis, Legendre polynomials, Walsh basis, prolate spheroidal functions and discrete cosine transform (DCT). Here the DCT basis is employed due to the fact that DCT is nearly optimal under the energy compaction criteria if the process is Markov (Eom 1999). It means using DCT basis allows for a lower expansion dimension of time-varying coefficients, i.e. resulting in a small value of $m$. DCT basis is of the following form,

$$
f_{j}(k)=a(k) \cos \left(\frac{\pi j(2 k+1)}{2 N}\right), a(k)=\sqrt{\frac{1}{N}}, k=0, a(k)=\sqrt{\frac{2}{N}}, k=1,2, \ldots, m
$$

\subsection{Identification of time-varying parameters}

Given the expansion by (3), the TVAR equation (1) can be rewritten as

$$
x(n)=-\sum_{i=1}^{p} \sum_{j=0}^{m} a_{i j}(n) f_{j}(n) x(n-i)+\sigma(n) .
$$

Define following vectors,

$$
\begin{aligned}
\mathbf{X}_{n}^{T}= & {\left[f_{0}(n) x(n-1), f_{1}(n) x(n-1), \ldots,\right.} \\
& \left.f_{m}(n) x(n-1)|\cdots| f_{0}(n) x(n-p), \ldots, f_{m}(n) x(n-p)\right] \\
\mathbf{A}= & {\left[a_{10}, a_{11}, \ldots, a_{1 m}|\cdots| a_{p 0}, a_{p 1}, \ldots, a_{p m}\right]^{T} . }
\end{aligned}
$$

Then Eq. (5) becomes

$$
x(n)=-\mathbf{X}_{n}^{T} \mathbf{A}+\sigma(n) .
$$

The estimation of time-varying parameters $\left\{f_{j}(n)\right\}$ thus converts into that of time invariant parameters A. From the view point of system identification, the terms $\mathbf{A}, X_{n}^{T}$ and $x(n)$ could be regarded as the system parameters, inputs and outputs of a system to be identified, respectively. In view of the superior performance of (ANNs) in system identification and prediction as well as its parallel computing, ANNs have been employed into the AR model identification in reference (Tian \& Juhola 1997). Here we extend this idea to the TVAR identification. The topology of the ANN used in the present study is depicted in figure 1. The ANN has two 


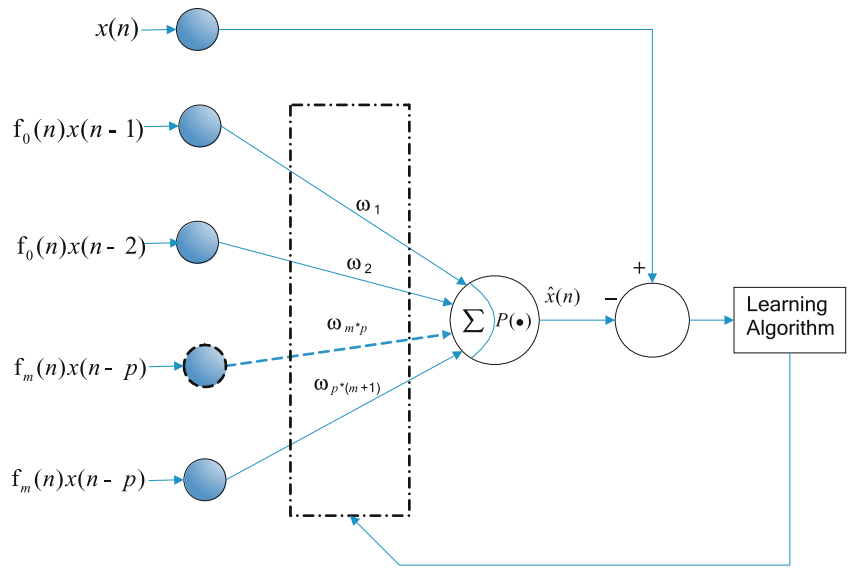

Figure 1. ANN used to estimate model parameters of TVAR.

layers, where the first layer receives the inputs $X_{n}^{T}$ and the second layer produce the estimated output $\hat{x}(n)$. The transfer function $p(\bullet)$ is a purely linear function.

$$
p(x)=x
$$

Consequently, the output of the ANN is

$$
\begin{aligned}
x(n) & =p\left(\omega_{1} f_{0}(n) x(n-1)+\omega_{2} f_{0}(n) x(n-2)+\cdots+\omega_{p(m+1)} f_{m}(n) x(n-p)\right) \\
& =\omega_{1} f_{0}(n) x(n-1)+\omega_{2} f_{0}(n) x(n-2)+\cdots+\omega_{p(m+1)} f_{m}(n) x(n-p) .
\end{aligned}
$$

Comparing Eq. (8) with Eq. (5), one will find that the model parameters $\mathbf{A}$ are equal to the weights $\omega$ which present the connection strength between input and output layer. The issue of TVAR model parameters identification thus turns into that of ANN learning. The well-known $\mathrm{BP}$ algorithm was used to train the ANN, aimed at finding proper weights $\omega$ to minimize the energy $E$.

$$
E=\frac{1}{2} \sum_{n=1}^{N} \sigma(n)^{2}=\frac{1}{2} \sum_{n=1}^{N}\left[x(n)-p\left(\mathbf{X}_{n}^{T}, \omega\right)\right]^{2} .
$$

The $\operatorname{grad}$ of $E$ is

$$
\operatorname{grad}_{\omega} E=\frac{\partial E}{\partial \omega}=-\sum_{n=1}^{N}\left[x(n)-p\left(\mathbf{X}_{n}^{T}, \omega\right)\right] \frac{\partial p\left(\mathbf{X}_{n}^{T}, \omega\right)}{\partial \omega} .
$$

Gradually correcting $\omega$ along the direction of negative gradient until to convergence, energy $E$ will arrive around its local minimums. Setting a step $\lambda$ and the initial value $\omega^{(0)}$, the correcting rule of $\omega$ is given by

$$
\omega=\omega^{(0)}-\lambda \operatorname{grad}_{\omega} E .
$$




\subsection{Determination of model order}

For accurate modelling of non-stationary signals, the model orders $p$ and $m$ should be correctly determined. At present, there are a lot of order determination methods for stationary AR and ARMA such as AIC, BIC FPE and MDL. But these approaches resort to exhaustive search and are designed only for stationary models. In the present work, a GA-based order search method is adopted due to the global search capability and higher search speed of GAs (Kalyanmoy 1999).

As depicted in figure 2, the GA-based order determination scheme consists of following steps (Vesin \& Grüter 1999; Ramaswamy 2006; Tomamaso \& Irene 2001; Chen 2005).

(i) Set a proper size for initial solutions with suitably encoding (conventionally called chromosomes) to the problem at hand.

(ii) Rank the solutions with an evaluation function (often called the fitness measure).

(iii) Create new solutions starting from existing ones by operators inspired by biology including selection, cross-over and mutation.

(iv) Create new population starting from the current one by an evolution strategy, i.e. to create successive generations of solutions.

(v) Stop the process by a termination criterion, usually based on a maximum number of generations or a threshold on the fitness measure.

Since $p$ and $m$ are integers, each solution (or chromosome) is denoted by a binary string. The crossover operator as in most GA implementations is used here, which build a new chromosome from its parents by concatenating randomly chosen tails of these two chromosomes. Mutation creates new chromosomes from existing ones by random alteration of some bits with a prescribed probability called the mutation rate.

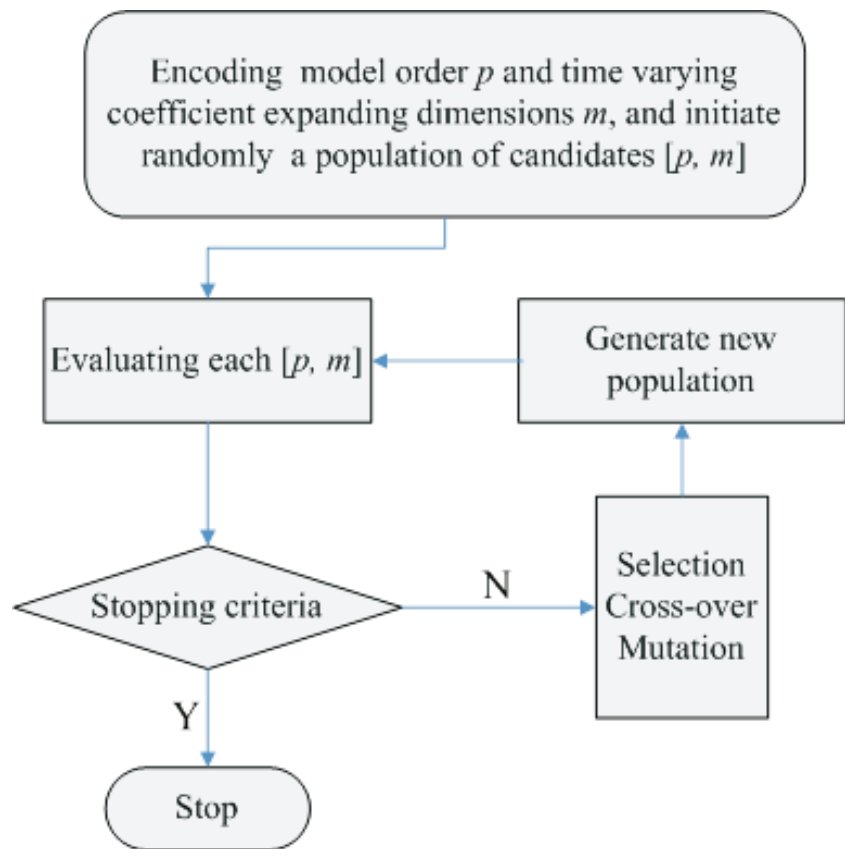

Figure 2. Flowchart of GA-based order determination in TVAR modelling. 
Eom (1999) proposed a maximum-likelihood decision rule for TVAR model order determination, which is as follows,

$$
J(p, m)=\frac{p(m+1)+2-N}{2} \ln (2 \pi \delta)-\frac{1}{2} \ln \left[\left|\sum_{n=p+1}^{N} X_{N}^{T} X_{N}\right|\right],
$$

where $\delta=\frac{1}{N-p} \sum_{n=p+1}^{N}(x(n)-\hat{x}(n))^{2}$.

The model order $p$ and $m$ are identified by maximizing the function $J(p, m)$ for different choices of $p$ and $m$. Here, the Eq. (12) is adopted as the evaluation function for the GA paradigm. The individual $[p, m]$ maximizing $J$ is the output of this order determination scheme. For employing TVAR to obtain a TFR of clear physical meaning, the values of $p$ and $m$ may need a fine-tuning around the ones obtained above.

\subsection{TVAR-based time frequency representation}

After time invariant parameters A in Eq. (6) have been estimated by ANN, time-varying coefficients $\left\{a_{i}(n), i=1,2, \ldots, p\right\}$ could be obtained from Eq. (3). Then time-frequency spectrum of TVAR model can be defined as (Conforto \& D'alessio 1999; Wang et al 2008; Poulimenos \& Fassios 2006; Sodsri 2003; Eom 1999)

$$
S(f, n)=\frac{\delta^{2}}{|A(z, n)|^{2}},
$$

where, $z=e^{j 2 \pi f}$

$$
\begin{aligned}
A(z, n) & =1+a_{1}(n) z^{-1}+\cdots+a_{p}(n) z^{-p} \\
\delta^{2} & =\frac{1}{N-p} \sum_{n=p+1}^{N} \varepsilon(n)^{2} .
\end{aligned}
$$

\section{Comparison of various TFR}

A rotor will pass through its sub-critical, critical and even super-critical speed during runup stage, hence much more information indicating the machine condition will be revealed in this process than at steady stage. Sekhar \& Prabhu (1998) and Behzad \& Ghias (2006) show the presence of a rotor crack would be more clearly indicated during run-up than at steady-state. So, in this section, a synthesized test signal, simulating the rotor vibration of run-up stage was analysed by various TFR, for the purpose of suggesting a recommendation to select an appropriate method for specific applications. The simulated signal is composed of three chirp components of which the two components of high frequency are spaced very close in time-frequency plane in order to simulate the parameter coupling between various mechanical parts. The last component is of amplitude modulation (AM) intended for imitating rotor vibration during passage through critical speed. The signal is given by the following equation,

$$
\begin{aligned}
& \left.x=\sin \left(2 \pi\left(f_{1}+a t\right) t\right)+\sin \left(2 \pi\left(f_{2}+a t\right) t\right)+\sin (2 \pi t) \cdot \sin \left(2 \pi\left(f_{3}+a t\right) t\right)\right) \\
& t=0: 0.001: 0.511 \\
& a=100, f_{1}=200, f_{2}=220, f_{3}=100 .
\end{aligned}
$$


The signal waveform accompanied by its three components and its Fourier spectrum are depicted in figure 3(a) and (b) respectively. According to the Fourier spectrum, it may be inferred that the frequency content of the signal covers roughly from $100 \mathrm{~Hz}$ to $320 \mathrm{~Hz}$, but the number of the components and how the frequency and amplitude change over time

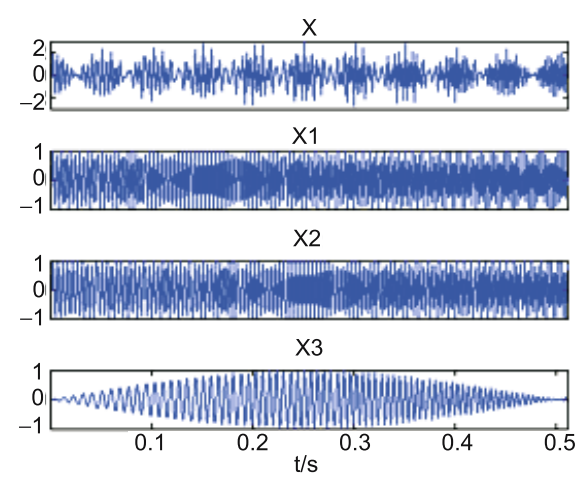

(a)

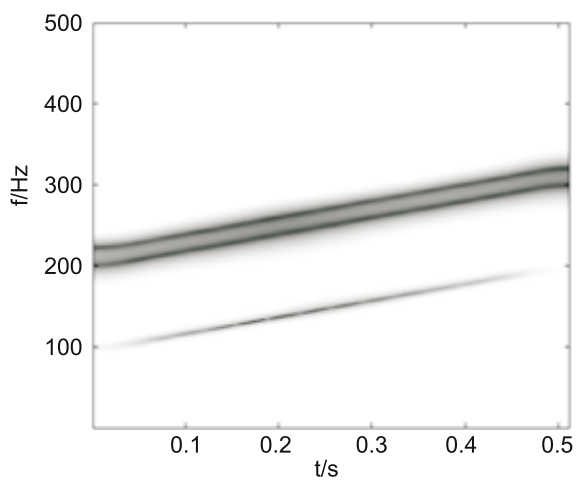

(c)

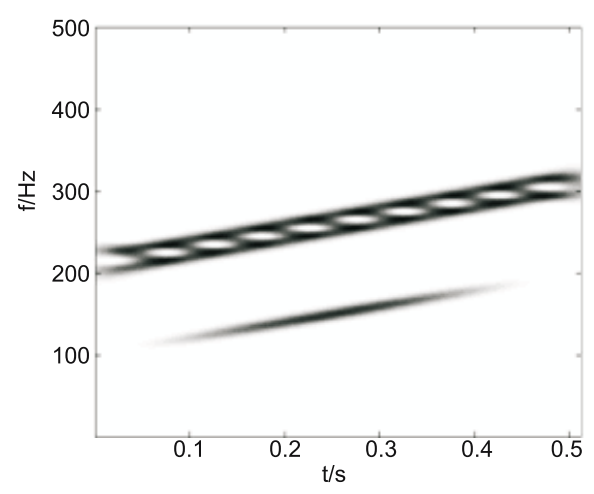

(e)

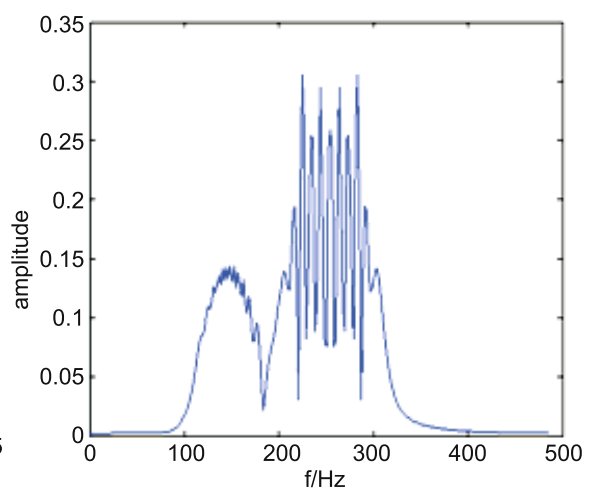

(b)

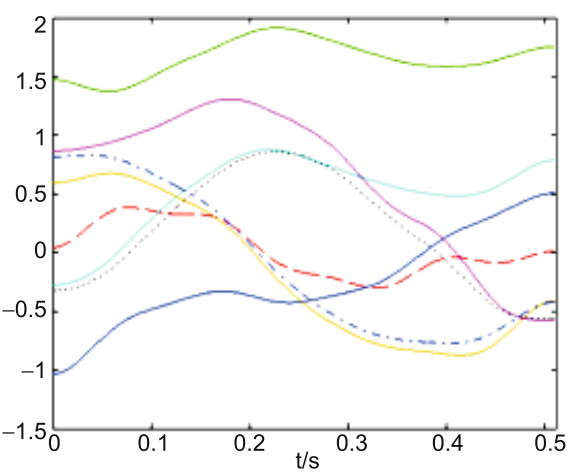

(d)

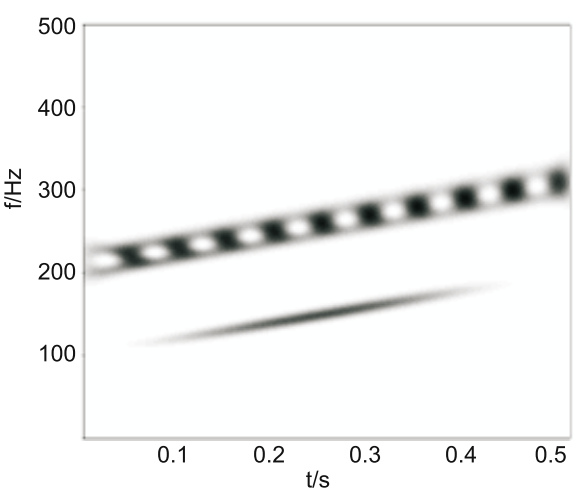

(f)

Figure 3. (Continued). 


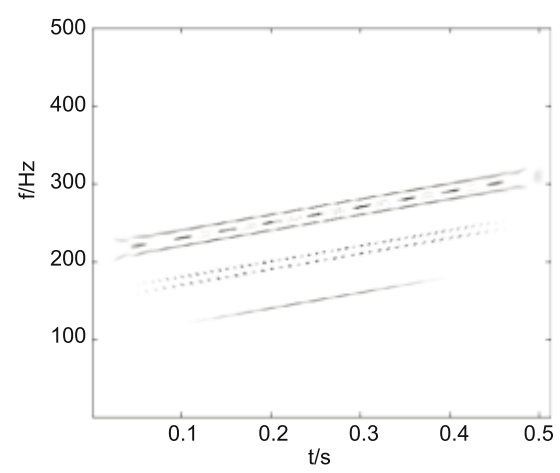

(g)

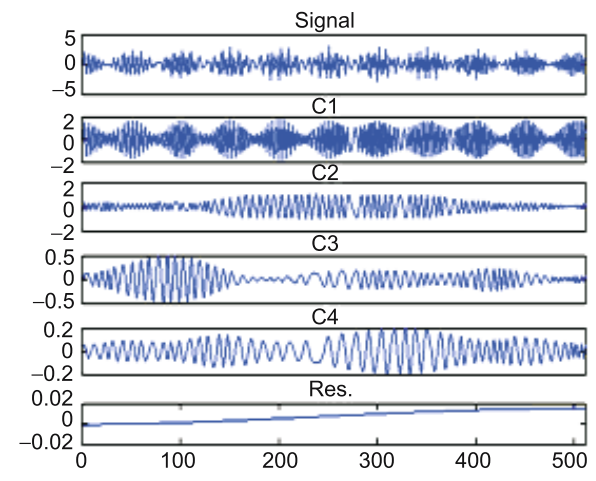

(I)

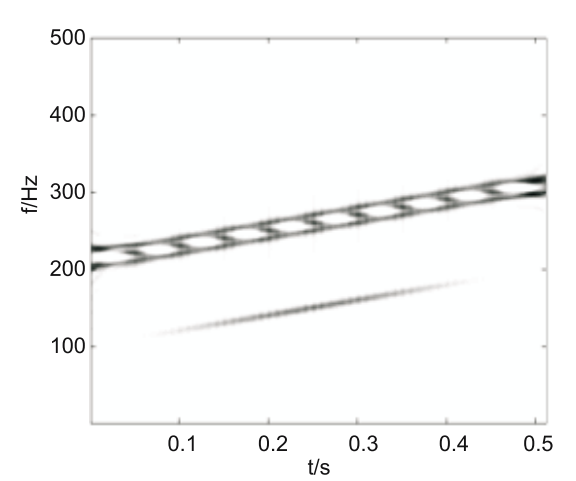

(h)

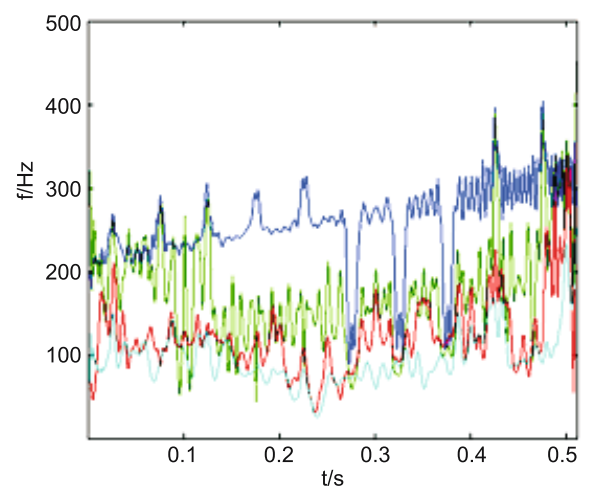

(j)

Figure 3. Synthesized signal and its three components (a), Fourier spectrum (b), TVAR (8, 10) based TFR (c), and its time-varying coefficients (d), TFR based on STFT with hamming windows of 128 samples long (e), CWT of complex morlet wavelet (f), WVD (g), and CWD (h), empirical model decomposition (EMD) (i), and corresponding instantaneous frequency (j).

are unknown. This is attributed to the fact that the FFT spectrum only gives an averaged result and cannot give information on time localization. Figures 3(c) and (d) are the TFR and time-varying coefficients of TVAR respectively. Here TVAR model of order $p=8, m=8$ is adopted by using the proposed order determination procedure. In figure 3(c), the $X$ axis represents time instants and $Y$ axis denotes frequency, and the colour level plotted in logarithmic scale indicates the power of signals. The following figures hold the same denotations. In figure 3(c) the two close spaced high frequency components are separated clearly, while the STFT (figure 3e) and the CWT (figure 3f) fail because of their poor time-frequency resolutions due to 'Uncertainty principle'. Here the hamming windows with a width of 128 samples and complex Morlet wavelet were employed in the STFT and CWT respectively, by using which the best performance was obtained for each of them. Figure 3(g) shows the WVD of the testing signal. The frequencies are concentrated on thin time-frequency lines indicating concentration of energy but the presence of strong cross-terms detrimentally compromise its success. Among various improved methods for suppressing cross-terms, CWD is most widely used (Rajagopalan et al 2005; Conforto \& D'alessio 1999). As shown in figure 3(h), the CWD suppresses the cross-terms of WVD greatly but, meanwhile the resolution degrades too much such that the two high frequency components cannot be discriminated. Another 


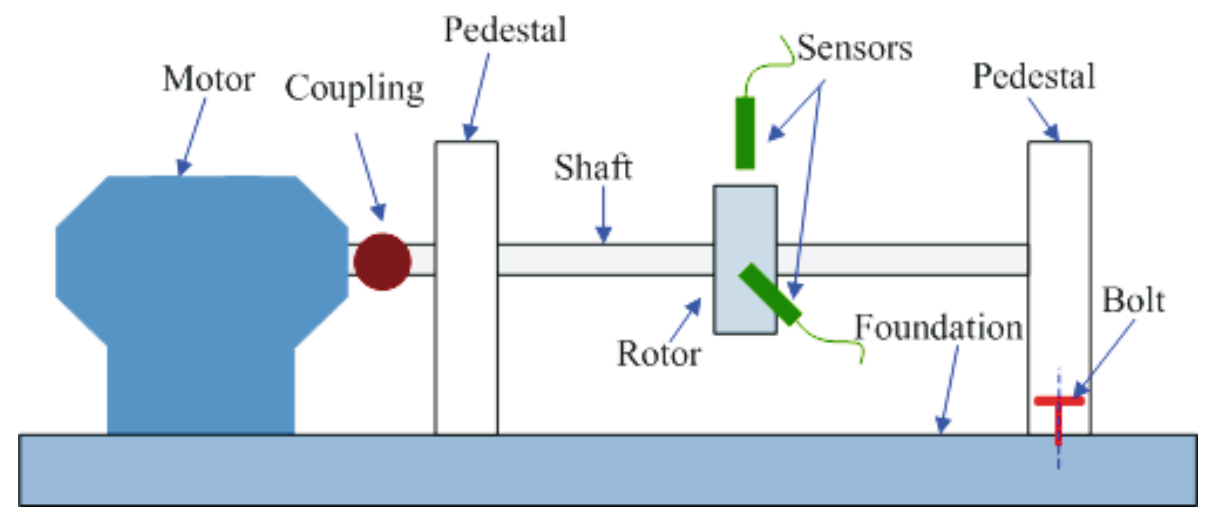

Figure 4. Experimental rig.

problem with the WVD and its derivations is their inability to resolve spectrum components at the endpoints of the analysed signal (Shan 1999). Figure 3(i) displays the first four IMFs containing the most proportion of the energy of the analysed signal as well as the residue of EMD decomposition. EMD cannot decompose the two higher frequency components and treats them as one component with amplitude modulation, even if very strict stop criteria and very large number of sifting step were used. The IMF C3 and C4 are not physically meaningful in comparison with the real components of the original signal. Accordingly, the instantaneous frequency-time curves shown in figure 3(j) do not make much physical sense.

\section{Experimental results}

To further examine the properties of TVAR, some experimental data were analysed. The experimental rig depicted in figure 4 consists of a rotor supported by two journal bearings and driven by a velocity controllable motor. Data were collected during run-up stages by using two non-contact eddy-current sensors, which measure the horizontal and vertical vibration of the rotor at a sampling frequency of $2,560 \mathrm{~Hz}$. Four kinds of condition were simulated including normal, unbalance, looseness and rub. Considering computational efficiency, only the STFT results were provided as a comparison benchmark for TVAR. The following timefrequency spectrums are shown in the frequency range from $0 \mathrm{~Hz}$ to $400 \mathrm{~Hz}$, since there is no useful information beyond this scope in our experiments.

\subsection{Normal}

Figure 5 shows the signal measured during the run-up stage when the rotor is in a normal condition. Figure 5(a) indicates rotor has not reached its resonance frequency. The Fourier spectrum in figure 5(b) spreads over a range roughly from $120 \mathrm{~Hz}$ to $170 \mathrm{~Hz}$, where the number of harmonic components and how their frequency and amplitude changes over time cannot be identified, which is often referred to as 'frequency smearing' in Fourier spectrum. In the STFT and TVAR time-frequency spectrum, one main frequency component corresponding to its rotating speed can be observed. This vibration component comes from the fact that a rotor will never be in an absolutely normal condition, i.e. inevitably original unbalance and bending will lead to this vibration component for almost all real rotor systems. Figure $5 \mathrm{c}$ and $\mathrm{d}$ 


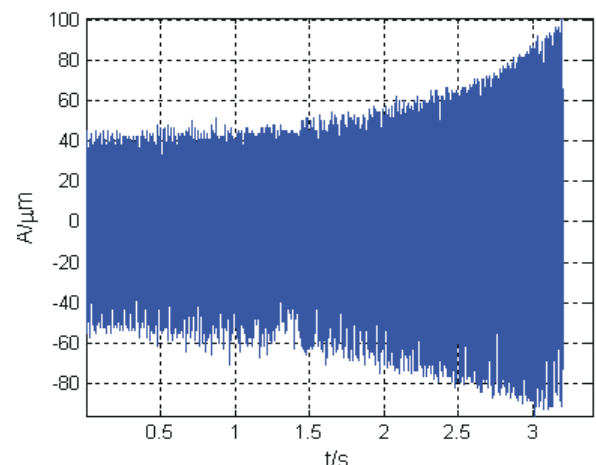

(a)

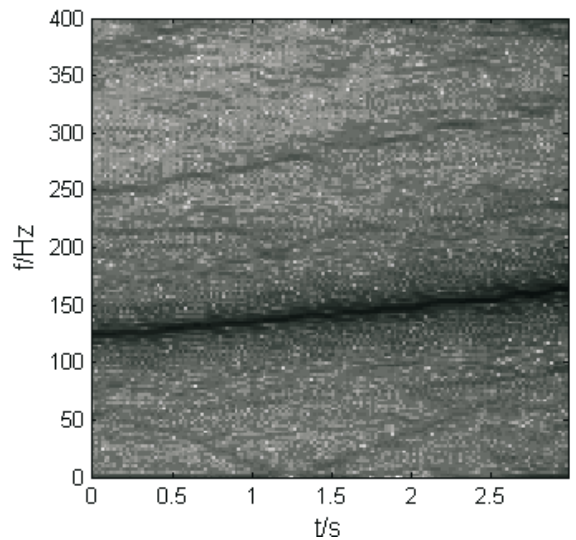

(c)

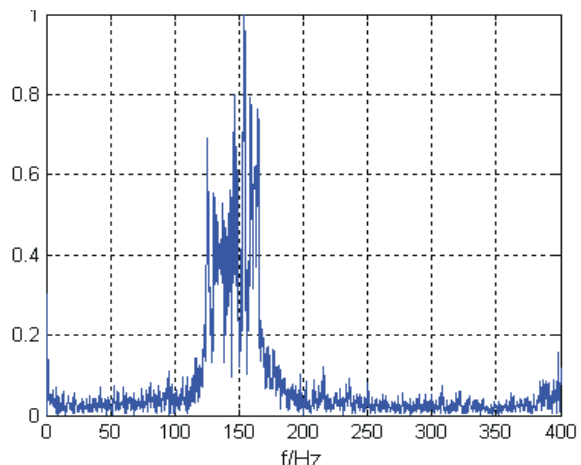

(b)

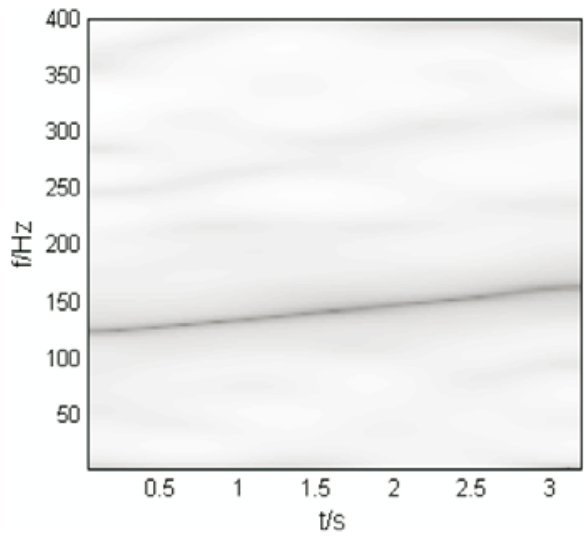

(d)

Figure 5. Signal collected under normal condition during run-up stage (a), Fourier spectrum (b), STFT (Kaiser window of 400 samples long and 300 samples overlapping) (c), TVAR of $p=30, m=5$ (d).

give similar results, indicating the time-frequency spectrum of TVAR can reveal correctly the property of the signal. The TVAR spectrum is clearer than that of the STFT, which means TVAR is more noise insensitive than STFT.

\subsection{Unbalance}

The results of unbalance are shown in figure 6. In addition to the first rotation frequency as in the normal condition, the second and weak third order harmonic frequency appear in figure $6 \mathrm{c}$ and d, which can be interpreted as the intrinsic nonlinearity of the rotor-bearing system. Again, the time-frequency spectrum of TVAR coincides with and is clearer than that of STFT.

\subsection{Looseness}

Figure 7 shows the results of the rotor vibration signal when a pedestal looseness fault in vertical direction is artificially introduced. This fault was simulated by loosening the bolt, by which the foundation is connected with the pedestal on the side far from the motor. The looseness fault would alter the stiffness of rotor system periodically in the presence of original unbalance or bending on the rotor, which will result in nonlinearity. Nonlinearities can lead 


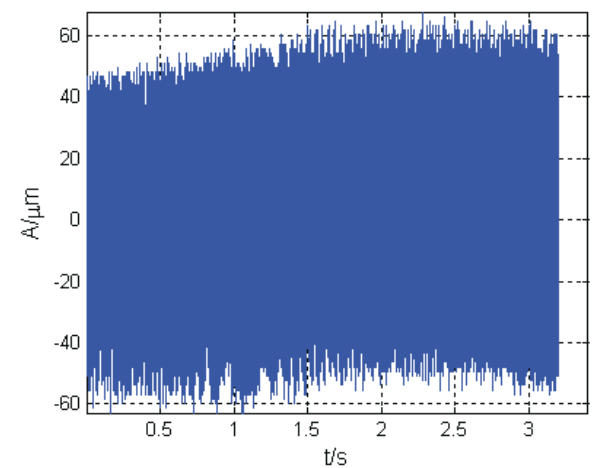

(a)

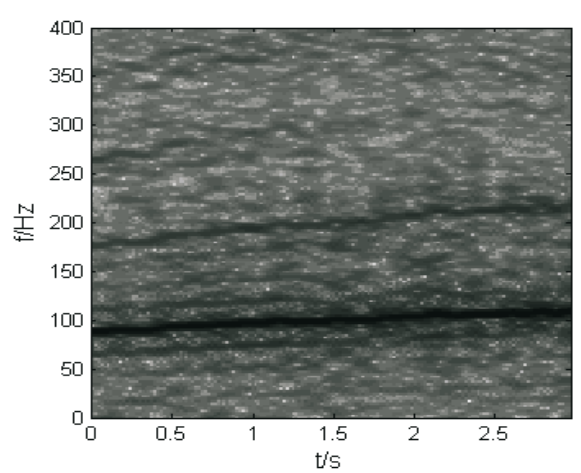

(c)

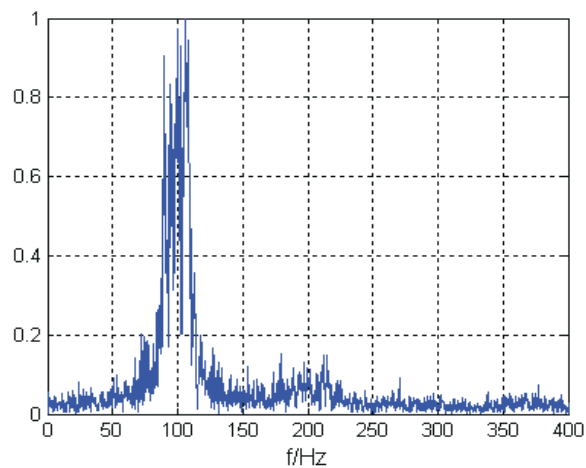

(b)

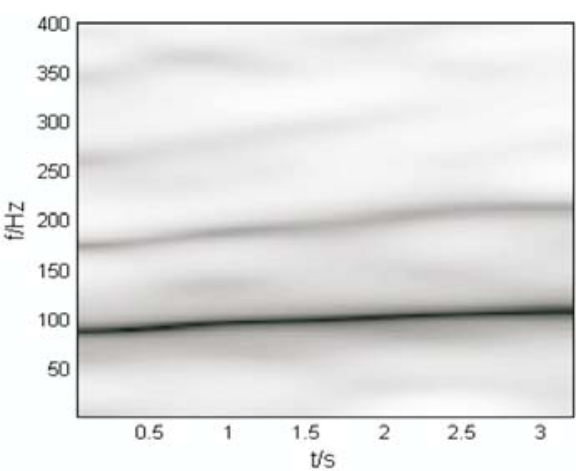

(d)

Figure 6. Signal collected under unbalance condition during run-up stage (a), Fourier spectrum (b), STFT (Kaiser window of 400 samples long and 300 samples overlapping) (c), TVAR of $p=30, m=5$ (d).

to more harmonic vibrations. In figure $7 \mathrm{c}$ and $\mathrm{d}$, besides the first rotation frequency, the second, third and forth order harmonic components come up. Compared with the normal and unbalance condition, looseness fault results in more numbers of harmonic components.

\section{$4.4 R u b$}

Figure 8 shows the results of a local rub fault. Local rub means that there is a contact between the rotor and the stator during part of one rotation of the rotor. Rubs between the surface of a rotor and the surface of a stator brings a time-varying stiffness to the rotor-bearings system. When the rotor and the stator come into contact with each other, the stiffness of the rotor-shaft will increase. Therefore, similar to the looseness fault, rub fault will bring nonlinearity to the system. In our experiment, the local rub fault was created by following steps. Firstly, a little mass was added to the disk to facilitate the occurrence of rub fault. Secondly, a brass screw was installed on the mounting block near the shaft, and keeps a small clearance between the shaft and brass screw. At last, we gradually increased the motor speed. Once the shaft and brass screw come into contact with each other, different level of rub fault can be achieved by continue to increase the motor speed. As a result, rub fault will manifest as some high order harmonic components in time-frequency spectrum. When rotor runs at a speed under 


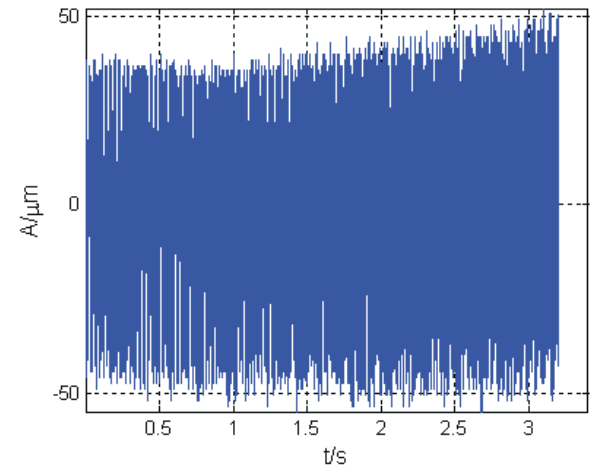

(a)

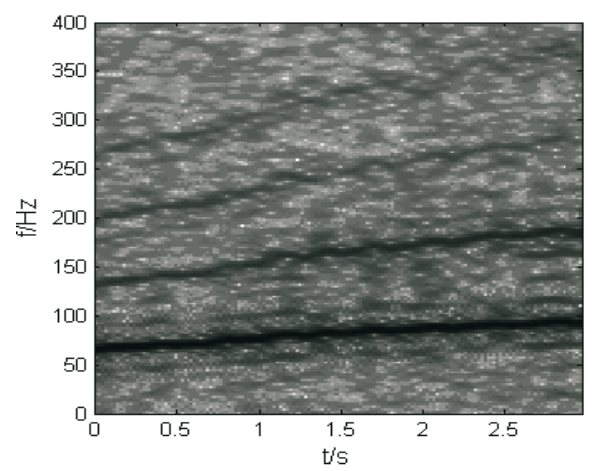

(c)

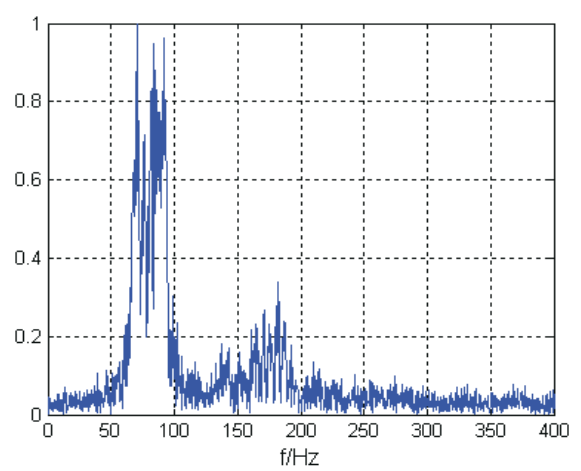

(b)

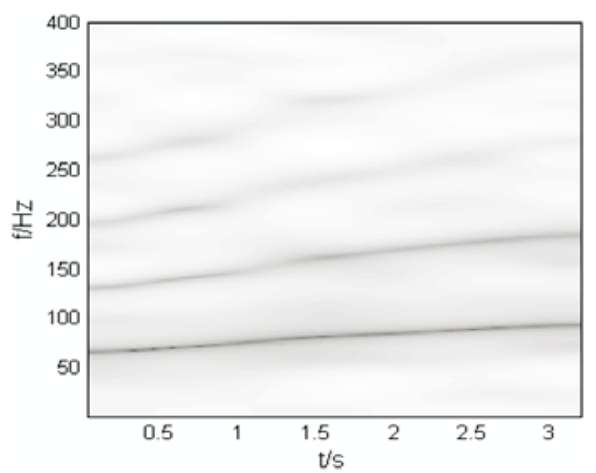

(d)

Figure 7. Signal collected under looseness condition during run-up stage (a), Fourier spectrum (b), STFT (Kaiser window of 400 samples long and 300 samples overlapping) (c), TVAR of $p=40, m=5(\mathbf{d})$.

the first order critical speed, the features of rub fault will be characterized by higher order harmonics (Liu \& Hu 2001). As shown in figure 8c and d, rub fault gives rise to the first six order harmonics in the time-frequency plane, which means the nonlinearity caused by the rub fault is more serious than that by the looseness fault which making only the first four orders of harmonic in our experiments. At the same time, only the first order component can be observed in the FFT spectrum shown in figure $8 \mathrm{~b}$.

Experimental rotor vibration signals of four kinds of faults were processed by the FFT, STFT and TVAR. Because of being collected during run-up stage, these signals possess non-stationary properties, i.e. frequency modulation and amplitude modulation. The Fourier spectra fails to characterize these signals, by which only one or at most two peaks can be identified, whereas much more harmonic components have been submerged in noise or obscured by the 'average' property of Fourier spectra. Limitations of Fourier analysis impel non-stationary signal processing techniques such as time-frequency analysis. The similarity between the results of STFT and TVAR indicate TVAR could correctly display the information of these non-stationary vibration signals. While compared with STFT, TVAR can identify the components of the signals much more clearly in the time-frequency spectrum, which demonstrates the noise resistance ability of TVAR. As different fault is expected to introduce different level of nonlinearity to systems, different fault will manifest as different 


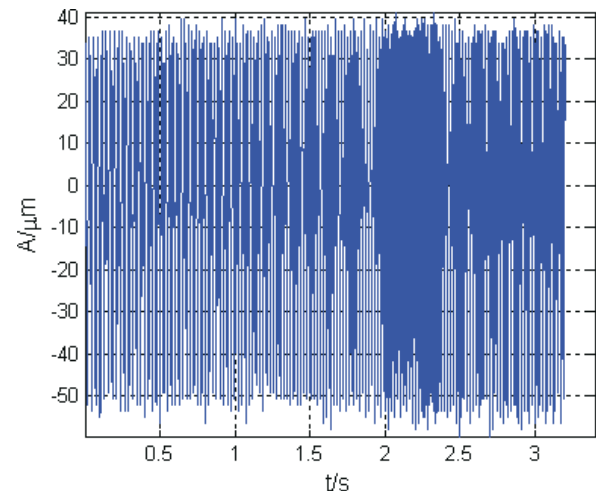

(a)

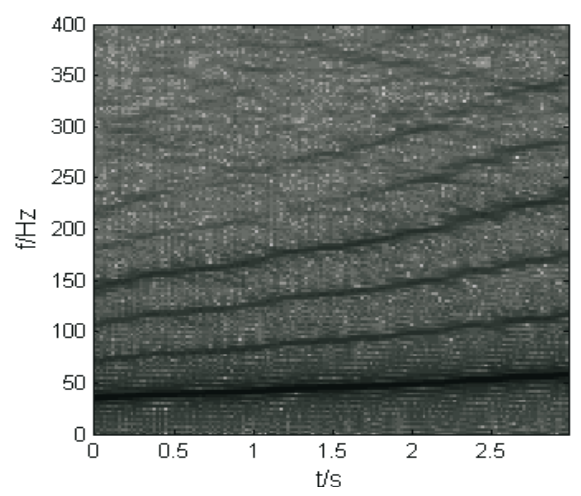

(c)

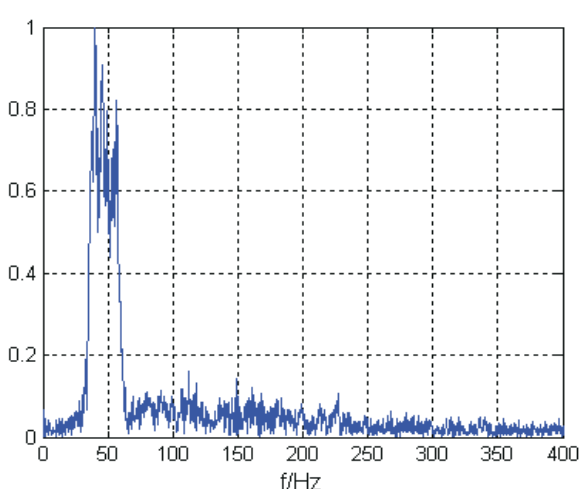

(b)

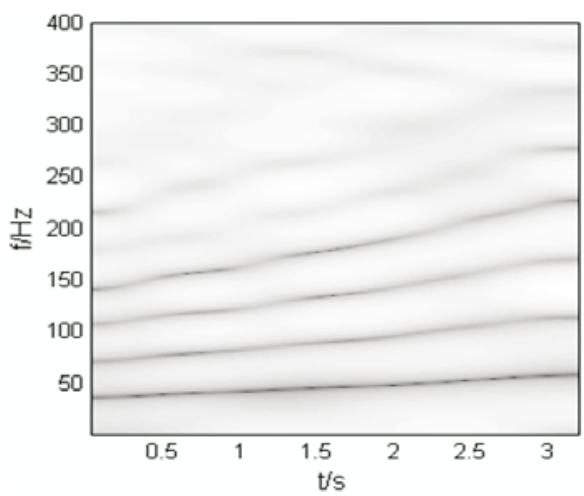

(d)

Figure 8. Signal collected in rub condition during run-up stage (a), Fourier spectrum (b), STFT (Kaiser window of 400 samples long and 300 samples overlapping) (c), TVAR of $p=40, m=5$ (d).

number of harmonic components in time-frequency spectrum as observed in figures $5 c-d$ and figures $8 \mathrm{c}-\mathrm{d}$.

\section{Conclusions}

A parametric TFR technique, known as time-varying autoregressive model has been presented. A basic function expansion policy and neural network-based time-varying coefficients identification are presended. Since the conventional criteria for model order selection are designed only for stationary AR or ARMA model, and there are totally two parameters including model order $p$ and time-varying coefficient expansion dimension $m$ involved in TVAR modelling, a genetic algorithm-based model order determination scheme along with a maximum likelihood rule has been presented. These proposals have been validated by numerical simulation and experiments. Simulation demonstrates TVAR is advantageous over some non-parametric TFRs such as STFT, CWT, WVD and EMD with respect to the resolution, accuracy, cross term as well as noise resistance. This conclusion has been further verified by experimental data collected from a rotor test rig during run-up stages, where four conditions including the normal, unbalance, looseness and rub were artificially induced. 
In the time-frequency spectrum of TVAR, such four faults can be visually discriminated clearly from each other with respect to the number of harmonics, which could be interpreted as different fault would introduce different level of nonlinearity to the systems. As scuh, it can be concluded that TFR based on TVAR could assist machine operators to diagnose and discriminate different faults.

This research was sponsored by the Natural Science Foundation of Jiangxi Province, China (Grant No. 0450017) and partially by the National Natural Science Foundation of China (Grant No. 50875161, 50821003). The authors would like to thank Dr. Yimin Zhan (University of Toronto) for communications with him on machinery condition monitoring under varying operating conditions. Authors would also like to thank the anonymous reviewers for their useful comments.

\section{References}

Behzad M, Ghias A 2006 Time-frequency feature extraction of a cracked shaft using an adaptive kernel. Appl. Mech. Mater. 5-6: 37-44

Chen $\mathrm{G} 2005$ New technique for determinating the order of ARMA model based on genetic algorithm. Chinese J. Mech. Eng. 41(1): 41-45 (in Chinese)

Chen Q, Huang N, Riemenschneider S, Xu Y 2006 A B-spline approach for empirical mode decompositions. Adv. Comput. Math. 24(1-4): 171-195

Chen Q, Worden K, Peng P, Leung A 2007 Genetic algorithm with an improved fitness function for (N)ARX modelling. Mech. Syst. Signal Proc. 21(2): 994-1007

Cheng J, Yu D, Yang Y 2006 Research on the intrinsic mode function (IMF) criterion in EMD method. Mech. Syst. Signal Proc. 20(4): 817-824

Cheng J, Yu D, Yang Y 2007 Application of support vector regression machines to the processing of end effects of Hilbert-Huang transform. Mech. Syst. Signal Proc. 21(3): 1197-1211

Chiementin X, Bolaers F, Dron J 2007 Early detection of fatigue damage on rolling element bearings using adapted wavelet. J. Vib. Acoust. 129(4): 495-506

Conforto S, D'alessio T 1999 Spectral analysis for non-stationary signals from mechanical measurements: A parametric approach. Mech. Syst. Signal Proc. 13(3): 395-441

Dowling M 1993 Application of non-stationary analysis to machinery monitoring. ICASSP-93 1: 59-62

Ebersbach S, Peng Z, Kessissoglou N 2006 The investigation of the condition and faults of a spur gearbox using vibration and wear debris analysis techniques. Wear 260(1-2): 16-24

Eom K 1999 Analysis of acoustic signatures from moving vehicles using time-varying autoregressive models. Multidim. Syst. Signal Proc. 10(4): 357-378

Gao R, Yan R 2006 Nonstationary signal processing for bearing health monitoring. Int. J. Manuf. Res. 1(1): $18-40$

Grenier Y 1983 Time-dependent ARMA modelling of non-stationary signals. IEEE T. Acoust. Speech Signal Proc. 31(4): 899-911

Gryllias K, Antoniadis I 2009 Selection of resonance bands in complex shifted Morlet wavelet (CSMW) based demodulation of defective rolling element bearings vibration response. Int. J. Wavelets Multi. 7(4): 387-410

Gupta K 1997 Vibration-A tool for machine diagnostics and condition monitoring. Sādhanā-(Acad. Proc. Eng. Sci.) 22(3): 393-410

Härmä A, Juntunen M, Kaipio J 2000 Time-varying autoregressive modelling of audio and speech signals. EUPSICO 2000: 2037-2040 
Hall M, Openheim A, Willsky A 1983 Time-varying parametric modelling of speech. Signal Process. 5(3): 267-285

Kalyanmoy D 1999 An introduction to genetic algorithms. Sādhanā-(Acad. Proc. Eng. Sci.) 24(4,5): 293-315

Li H, Yang L, Huang D 2005 The study of the intermittency test filtering character of Hilbert-Huang transform. Math. Comput. Simulation 70(1): 22-32

Li L, Qu L, Liao X 2007 Haar wavelet for machine fault diagnosis. Mech. Syst. Signal Proc. 21(4): $1773-1786$

Liu Y, Hu N 2001 Some observations of rub-impact fault on jeffcott rotor. J. Vib. Eng. 14(1): 96-99 (in Chinese)

Loutridis S 2006 Instantaneous energy density as a feature for gear fault detection. Mech. Syst. Signal Proc. 20(5): 1239-1253

Nagaraju C, Narayana Rao K, Mallikarjuna Rao K 2009 Application of 3D transforms in rotor systems. Sādhanā-(Acad. Proc. Eng. Sci.) 34(3): 407-419

Paya B, Esat I, Badi M 1997 Artificial neural network based fault diagnostics of rotating machinery using wavelet transforms as a preprocessor. Mech. Syst. Signal Proc. 11(5): 751-765

Peng Z, Chu F 2004 Application of the wavelet transform in machine condition monitoring and fault diagnostics: a review with bibliography. Mech. Syst. Signal Proc. 18(2): 199-221

Peng, Z, Tse W, Chu F 2005a An improved Hilbert-Huang transform and its application in vibration signal analysis. J. Sound Vib. 286(1-2): 187-205

Peng, Z, Tse W, Chu F 2005b A comparison study of improved Hilbert-Huang transform and wavelet transform: Application to fault diagnosis for rolling bearing. Mech. Syst. Signal Proc. 19(5): 974-988

Peng Z, Jackson M, Rongong J, Chu F, Parkin R 2009 On the energy leakage of discrete wavelet transform. Mech. Syst. Signal Proc. 23(2): 330-343

Poulimenos A, Fassois S 2006 Parametric time-domain methods for non-stationary random vibration modelling and analysis - A critical survey and comparison. Mech. Syst. Signal Proc. 20(4): $763-816$

Qi K, He Z, Zi Y 2007 Cosine window-based boundary processing method for EMD and its application in rubbing fault diagnosis. Mech. Syst. Signal Proc. 21(7): 2750-2760

Rai V, Mohanty A 2007 Bearing fault diagnosis using FFT of intrinsic mode functions in HilbertHuang transform. Mech. Syst. Signal Proc. 21(6): 2607-2615

Rajagopalan S, Restrepo J, Aller J, Habetler T, Harley R 2005 Selecting time-frequency representations for detecting rotor faults in BLDC motor operating under rapidly varying operating conditions. IECON: 2585-2590

Ramaswamy P 2006 Towards optimal model order selection for autoregressive spectral analysis of mental tasks using genetic algorithm. Int. J. Comp. Sci. Netw. Secur. 6(1): 153-162

Rouillard V, Sek M 2005 The use of intrinsic mode functions to characterize shock and vibration in the distribution environment. Packaging. Technol. Sci. 18(1): 39-51

Rubini R, Meneghetti U 2001 Application of the envelope and wavelet transform analysis for the diagnosis of incipient faults in ball bearings. Meach. Syst. Signal Pr. 15(2): 287-302

Sekhar A, Prabhu B 1998 Condition monitoring of cracked rotors through transient response. Mech. Mach. Theory. 33(8): 1167-1175

Shan P 1999 Time-varying autoregressive model based signal processing with applications to interference rejection in spread spectrum communications. Phd dissertation submitted to Virginia Polytechnic Institute and State University, Blacksburg

Shi D, Wang W, Qu L 2004 Defect detection for bearings using envelope spectra of wavelet transform. J. Vib. Acoust. 126(4): 567-573

Sodsri C 2003 Time-varying autoregressive models for non-stationary acoustic signal and its frequency analysis. Phd dissertation submitted to Pennsylvania State University, Pennsylvania

Tian J, Juhola M, Gronfors T 1997 AR parameter estimation by a feed back neural network. Comput. Stat. Data Analysis 25(1): 17-24 
Tommaso M, Irene P 2001 Building ARMA models with genetic algorithms. LNCS 2037: 335-342

Vesin J, Grüter R 1999 Model selection using a simplex reproduction genetic algorithm. Signal Process. 78(3): 321-327

Wang W, McFadden P 1996 Application of wavelets to gearbox vibration signals for fault detection. J. Sound Vib. 23(5): 927-939

Wang Y, Kootsookos P 1998 Modelling of low shaft speed bearing faults for condition monitoring. Mech. Syst. Signal Proc. 12(3): 415-426

Wang G, Luo Z, Qin X, Leng Y, Wang T 2008 Fault identification and classification of rolling element bearing based on time-varying autoregressive spectrum. Mech. Syst. Signal Proc. 22(4): 934-947

Zhan Y, Jardine A 2005 Adaptive autoregressive modelling of non-stationary vibration signals under distinct gear states. Part 1: modelling. J. Sound Vib. 286(3): 429-450

Zhan Y, Makis V, Jardine A 2006 Adaptive state detection of gearboxes under varying load conditions based on parametric modelling. Mech. Syst. Signal Proc. 20(1): 188-221 